W. V. Dörffel ${ }^{1}$
Y. T. Sugano
D. Stalling
L. Coconu $^{3}$
D. Hentschel
'
G. Linß
Ch. Witt

\section{Lasergestützte endoskopische Vermessung der großen Atemwege}

\author{
Laser-based Endoscopic Measurement of Airway Dimensions
}

\section{Zusammenfassung}

Die bronchoskopische Vermessung von Stenosen der großen Atemwege ist bisher nicht zufriedenstellend gelöst. Wir möchten im Folgenden unsere Methodik und die Ergebnisse der lasergestützten endoskopischen Vermessung von Atemwegsquerschnitten vorstellen. Neben der Bestimmung von Atemwegsquerschnitten kann mithilfe dieser Methode ein dreidimensionaler Datensatz rekonstruiert und visualisiert werden. Zur Bestimmung der Querschnittsflächen wird auf die Bronchialwand ein Laserlichtring projiziert. Der Laserlichtring kann im bronchoskopischen Bild mithilfe einer speziell entwickelten Software online segmentiert und vermessen werden. Die durch das Weitwinkelobjektiv bedingte Verzerrung des endoskopischen Bildes wird durch einen Bildverarbeitungsalgorithmus ausgeglichen. Das System wurde an Plastikröhrchen mit bekannten internen Diametern und an Schweineluftröhren getestet. Die realen Querschnittsflächen der Schweineluftröhren wurden nach der Endoskopie anhand von Trachealschnitten unter Zuhilfenahme einer verzerrungsfreien Video-Makro-Zoom-Linse bestimmt. Die Ergebnisse der Korrelation für die Plastikröhrchen $(r=0,99$, $\mathrm{p}<0,01$ ) waren geringfügig besser als der Vergleich der Querschnittsflächen von Schweineluftröhren $(r=0,88, p<0,01)$. Durch sequenzielle Aufnahme von Atemwegsquerschnitten konnten dreidimensionale Datensätze rekonstruiert und visualisiert werden. Die Ergebnisse unserer Untersuchungen demons-

\section{Abstract}

Bronchoscopy is the technique of choice for the evaluation of a stenosis in the large airways. However, no system has been successfully employed for the bronchoscopic measurement of airway stenosis. The purpose of these study was the development and validation of a method for measuring the cross-sectional areas in the large airways. Furthermore, this application should be used for the 3D-reconstruction and visualisation of airway stenosis. A laser probe inserted into the operating channel of the bronchoscope enabled assessment of the distance between the images and the tip of the bronchoscope by means of projecting a ring of light on to the endoluminal wall. Image distortion due to the wide-angle lens was corrected by a computer program developed by us. Plastic tubes with known diameters were used for validation. Additionally, distortion-corrected bronchoscopic images were compared with distortion-free videoscopic image analysis of tracheal slices taken from pigs. When plastic tubes were used, the correlation coefficient ( $r$ ) was slightly higher $(r=0,99, p<0,01)$ than the correlation of cross-sectional areas between bronchoscopic and videoscopic images of tracheal slices $(r=0,88, p<0,01)$. Furthermore the system could be used in a few patients for 2D and 3D measurement and visualisation of airway stenosis. Application of the present method offer quantitative assessment of airway stenosis located in the large airways. 
trieren, dass das System der lasergestützten endoskopischen Vermessung mit guter Genauigkeit zur bronchoskopischen Bestimmung von Atemwegsquerschnitten einsetzbar ist.

\section{Einleitung}

Die endoskopische Untersuchung gilt heute als das Verfahren der Wahl zur morphologischen Beurteilung von Stenosen der großen Atemwege. $\mathrm{Zu}$ den Informationen, die derzeit mit der Bronchoskopie verlässlich gewonnen werden können, zählen u.a. die Konfiguration einer Stenosierung, die Schleimhautbeschaffenheit und ggf. die Tumorausdehnung. Darüber hinaus sind die Blutungsbereitschaft der Läsion, eine möglicherweise bestehende Sekretretention und die Entfernung der Stenose von den Stimmbändern und den distal gelegenen Ostien für die Interventionsplanung von Bedeutung. Ein bronchoskopisches Verfahren zur Vermessung von Stenosen ist bisher nicht existent. Methodologisch sieht sich der Endoskopiker zwei Problemen gegenüber, die eine Lösung erfordern. Zum einen werden die endoskopischen Bilder durch das an der Spitze des Endoskops befindliche Weitwinkelobjektiv verzerrt. Dieser „Fish eye view“ bedingt, dass Objekte im Zentrum des Bildes größer zur Darstellung gelangen, peripher gelegene Strukturen des Objektes werden im Bild verkleinert abgebildet (Abb.1). Andererseits stellt das bronchoskopische Bild ein Summationsbild vieler Querschnitte des Tracheobronchialsystems dar. Zur Vermessung einer bronchialen Querschnittsfläche als Grundlage der Morphometrie einer Stenose ist es deshalb erforderlich, den Abstand zwischen Objektiv und der zu vermessenden Zirkumferenz zu bestimmen. Ziel dieser Untersuchung war die Entwicklung und Validierung eines endoskopischen Verfahrens zur Morphometrie von Atemwegsstenosen.

\section{Methoden}

Die Methode zur Bestimmung von Atemwegsquerschnitten umfasst eine Kombination der lasergestützten Entfernungsmessung mit einer Bildbearbeitung zur automatischen Identifizierung des Lichtrings und anschließender Korrektur der durch die Weitwinkeloptik hervorgerufenen Verzerrungen.

\section{Bronchoskopiesystem und Lasersonde}

Wir verwendeten für die Untersuchung das elektronische Bronchoskop BF 1 T 200 (Olympus Optical Co. Ltd., Tokyo, Japan) und den dazugehörigen Prozessor EVIS-CV 200 (Olympus). Mithilfe einer handelsüblichen Videokarte (Haupauge WinTV Go) wurden die endoskopischen Bilder in einen PC eingespeist und digitalisiert.

Die Messung des Abstandes zwischen Objekt (interessierender Atemwegsquerschnitt) und Objektiv des Endoskops wurde durch die Applikation von strukturiertem Laserlicht auf die Bronchialwand realisiert. Hierzu bedienten wir uns einer Laserfaser, die über den Arbeitskanal des Endoskops vorgeschoben wird. Wir verwenden eine HCS(hard cladding silica)-Faser mit einem Kerndurchmesser von $400 \mu \mathrm{m}$ und einer numerischen Apertur von

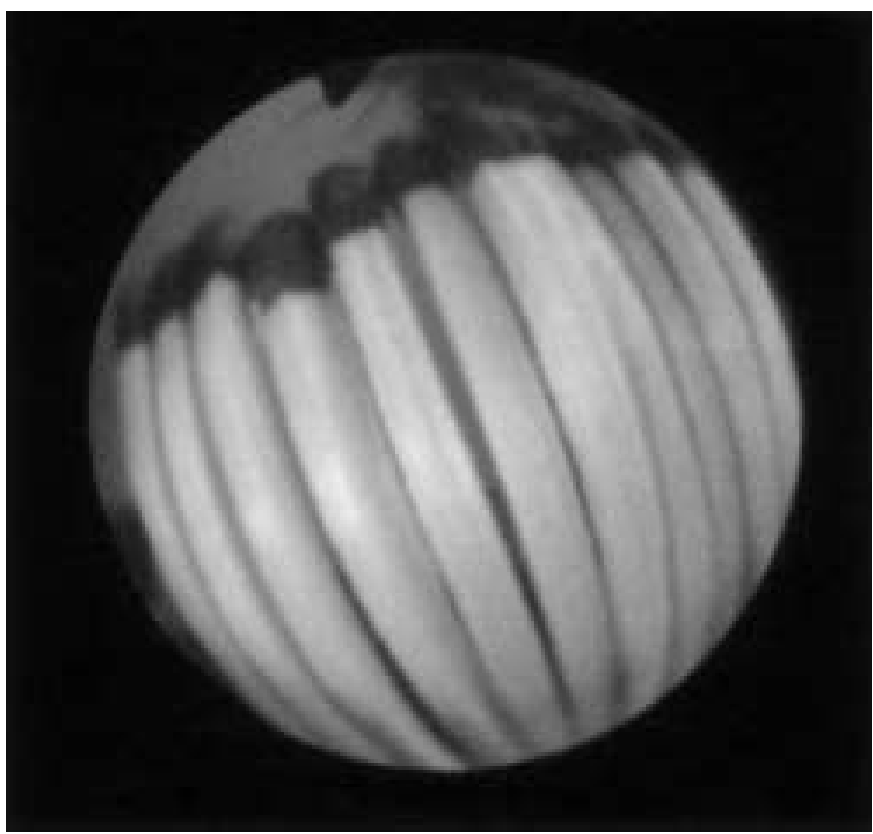

Abb. 1 Verzerrung bronchoskopischer Bilder. Strukturen in der Peripherie des Bildes werden kleiner als zentral gelegene Objekte abgebildet.

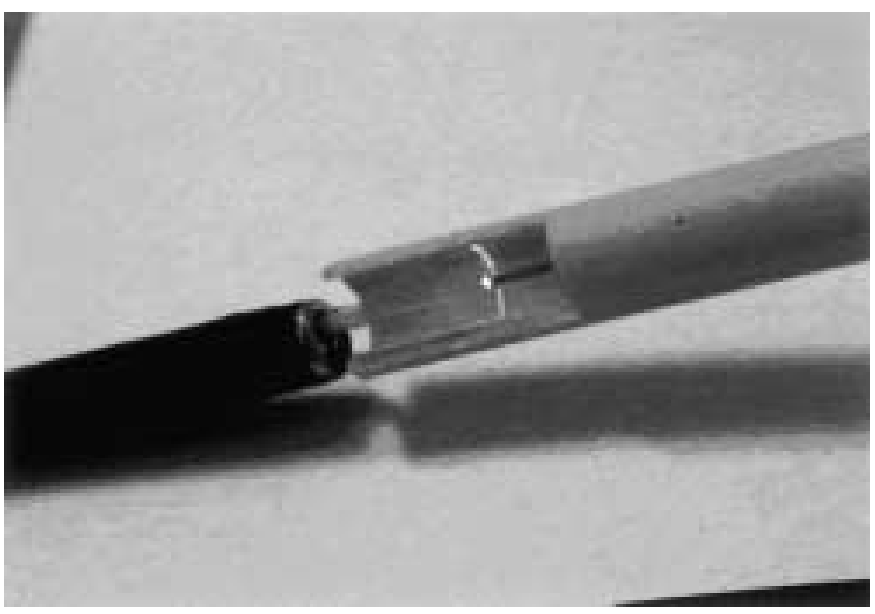

Abb. 2 Bronchoskop mit Lasersonde. Lichtleiter und Reflektor sind über einen transparenten Katheter verbunden. Dieser gestattet den Austritt des Laserlichtes in rechtem Winkel zur optischen Achse des Systems. Der Laserlichtring wird auf der Wand eines Testobjekts reflektiert.

0,18. Als Lichtquelle dient der Helium/Neon-Pilotstrahl eines Neodym-YAG-Lasers mit einer Ausgangsleistung von $1 \mathrm{~mW}$. Das Laserlicht wird über die Glasfaser auf einen Strahlenteiler geleitet, der das Licht im rechten Winkel auf die Bronchialwand projiziert (Abb. 2). Durch die Reflexion des Lichtes entsteht eine Ebene aus Licht, deren äußere Begrenzung durch einen Ring auf der Bronchialwand gebildet wird. Es hat sich als zweckmäßig erwiesen, die Umlenkung der Laserstrahlen in einer Entfernung von $15 \mathrm{~mm}$ zur Endoskopspitze zu realisieren. Da die Lasersonde im Arbeitskanal in Längsrichtung frei beweglich ist, ist es erforderlich, einen konstanten Abstand zwischen Reflektor und Objektiv zu gewährleisten. Dafür dient bei der Bronchoskopie eine Markierung auf der Lasersonde, die während der Untersuchung sichtbar ist. Eine Veränderung des Abstandes zwischen Reflektor 
und Objektiv äußert sich somit in einer veränderten Abbildung der Markierung im bronchoskopischen Bild und kann vom Untersucher rasch korrigiert werden. Bei zu kleinem Abstand $(<15 \mathrm{~mm})$ wäre ein größerer Messwert, bei zu großem Abstand ein kleinerer Messwert der Querschnittsfläche die Folge. Zwischen dem distalen Ende der Laserfaser und der Spitze des Strahlenteilers besteht eine Distanz von 0,5 mm. Beide sind über einen transparenten Katheter miteinander verbunden.

\section{Bildauswertung und Software}

Da die Entfernung zwischen Objektiv und Reflektor bekannt ist, ist es möglich, die Querschnittsfläche des Lichtrings zu bestimmen. Dazu wird der Lichtring zunächst automatisch mithilfe einer von uns entwickelten Software (Endo 3D) segmentiert und anschließend entzerrt. Für die Segmentierung verwenden wir einen strahlbasierten Algorithmus. Dabei gibt der Benutzer initial einen Punkt in der Bildmitte vor. Ausgehend von diesem Punkt werden Strahlen nach außen verfolgt und der jeweils hellste Punkt auf einem Strahl gesucht. Die Verbindung zwischen allen hellsten Punkten ergibt eine Approximation des Lichtrings. Durch verschiedene Filtertechniken wird erreicht, dass Rauschartefakte und Ausreißer weitgehend eliminiert werden. Bedingt durch die Bauart des Endoskops wird ein kleiner Teil des Lichtrings vom Lichtwellenleiter verdeckt. Der verdeckte Bildteil ist fix und kann vor der Untersuchung vom Benutzer ausmaskiert werden. In diesem Bereich werden keine Strahlen verfolgt. Stattdessen wird der Lichtring vom Mittelpunkt aus kreisförmig interpoliert. Der implementierte Algorithmus arbeitet zuverlässig, sofern geeignete Lichtverhältnisse vorherrschen (hoher Kontrast, Verzicht auf zusätzliche Beleuchtung) und sofern der Lichtring vollständig abgebildet wird. Zur Entzerrung der Bilder muss das Kamerasystem einmalig vermessen werden. Dazu wird auf einer optischen Bank ein Millimeterraster in der Lichtringebene positioniert. Das entsprechende Videobild wird wiederum eingelesen und digitalisiert. Anschließend klickt der Benutzer nacheinander die Kreuzungspunkte des Rasters an. Dadurch wird ein kartesisches Kontrollgitter definiert. Um die unverzerrten Koordinaten für einen beliebigen Bildpunkt zu erhalten, wird zunächst diejenige Zelle des Kontrollgitters gesucht, die den Bild-

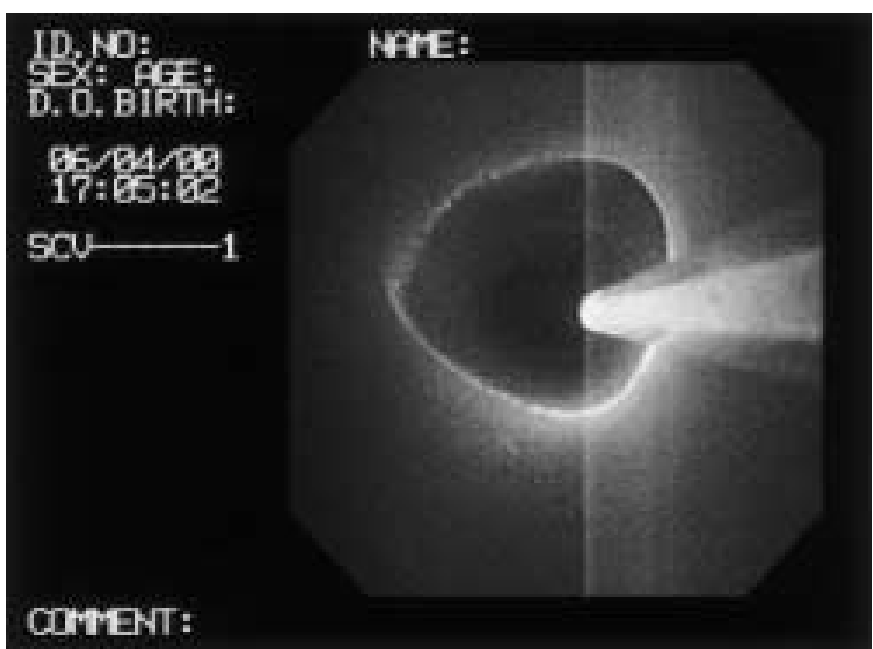

Abb. 3 Bronchoskopisches Bild der Trachea. Lasersonde mit Laserlichtring sind gut erkennbar. Der durch die Lasersonde verdeckte Teil des Laserlichtringes muss für die Berechnungen extrapoliert werden.

punkt enthält. Anschließend wird innerhalb der Zelle bilinear interpoliert. Außerhalb des Kontrollgitters wird entsprechend extrapoliert. Diese Art der Entzerrung ist sehr robust und in der Lage, beliebige nicht-lineare oder auch asymmetrische Linsenfehler zu kompensieren.

Nachdem das Kamerasystems kalibriert ist und der Mittelpunkt für die Strahlverfolgung definiert wurde, erfolgen alle weiteren Berechnungen in Echtzeit. Während der Untersuchung werden die aktuellen Videobilder zusammen mit dem segmentierten Lichtring und der dazugehörigen Querschnittsfläche am Monitor angezeigt (Abb. 3, u. 4).

\section{Durchgeführte Messungen}

Zuerst wurde das System an Kunststoffröhrchen und Modellen des Tracheobronchialsystems erprobt. Der bekannte Durchmesser der kreisrunden, weißen, aus Polystyrol bestehenden Kunststoffröhrchen betrug 6,2-17,2 mm.
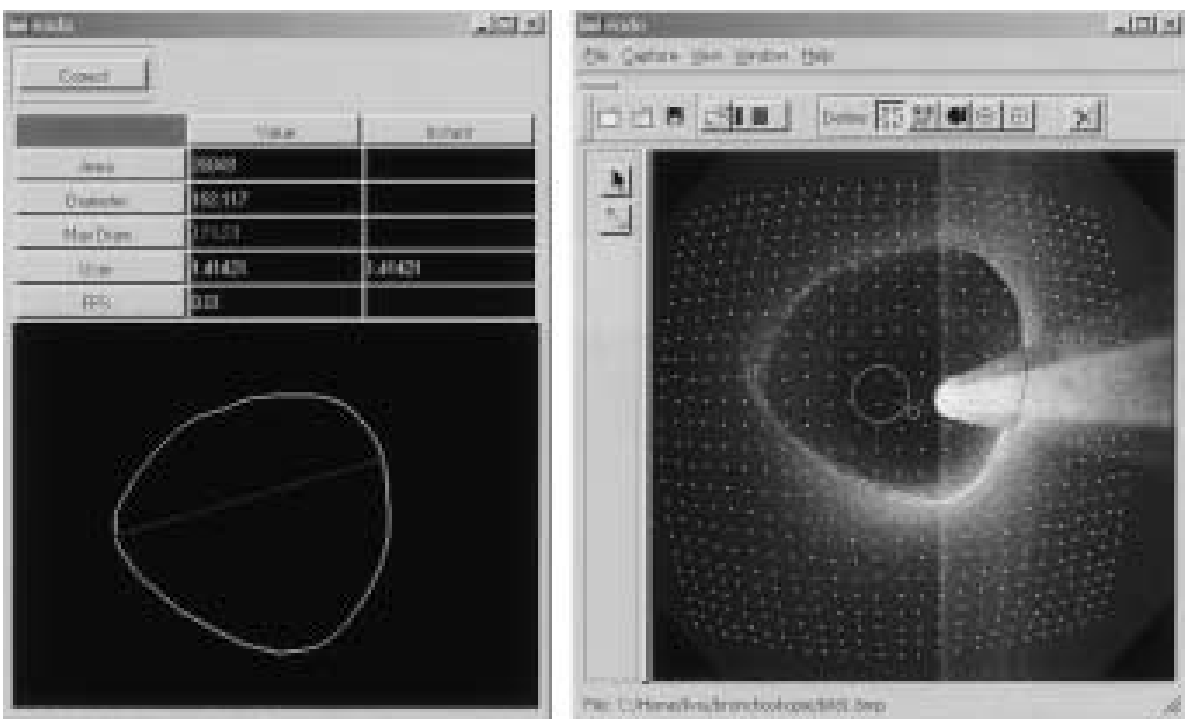

Abb. 4 Benutzeroberfläche des Programms zur Segmentierung und OnlineVermessung des Laserlichtringes. Rechts im Bild sind der segmentierte Laserlichtring und die Projektion der Koordinatenmatrix zum Ausgleich der Bildverzerrung erkennbar. Links wird der segmentierte Laserlichtring mit Angabe von Flächeninhalt und Umfang online abgebildet. 
In einem zweiten Schritt erfolgte die Validierung der Methodik an der Schweinetrachea. Die Trachea vom Schwein wurde konserviert (10\%iges Formalin) und die Form durch Plastinierung stabilisiert. Zunächst wurden die Schweineluftröhren mit der o.g. Methodik endoskopiert und Bilder im Abstand von $2 \mathrm{~mm}$ aufgenommen. Danach wurde die Trachea in $2 \mathrm{~mm}$ breite Ringe zerschnitten. Die Bestimmung der Querschnittsflächen der Trachealringe erfolgte mit Hilfe einer verzerrungsfreien Video-Makro-Zoom-Linse (18...108 mm, Fuji Photo Film Co. Ltd., Tokyo, Japan) und einer digitalen Kamera (WVE 550 3-IT-CCD, Panasonic, Matsushita Electric Industrial Co. Ltd., Osaka, Japan).

Zur Bestimmung der Inter- und Intraobservervariabilität wurden die Messungen von drei Untersuchern durchgeführt und von jedem Untersucher jeweils einmal wiederholt.

\section{3-D-Messsystem}

Für die Aufnahme eines dreidimensionalen Datensatzes erfolgte die Endoskopie der zentralen Atemwege mit der Aufnahme sequenzieller bronchoskopischer Bilder im Abstand von $1 \mathrm{~mm}$. Der Endoskopvorschub wurde mithilfe eines von der Fa. Rüsch konstruierten Applikationskatheters kontrolliert. Die mit dem oben beschriebenen Verfahren segmentierten und entzerrten Lichtring-Konturen wurden einzeln exportiert und mithilfe der Visualisierungssoftware Amira (www.amiravis.com) zu einem 3D-Modell zusammengesetzt.

\section{Statistik}

Die Korrelation zwischen den experimentell ermittelten Flächeninhalten und den tatsächlichen Flächen wurde mithilfe der linearen Regression untersucht (Pearson-Korrelationskoeffizient r, slope, intercept). Die Charakterisierung der Inter- und Intraobservervariabilität erfolgte mithilfe des Intraclass-Korrelationskoeffiezienten (ICC).

\section{Ergebnisse}

Die über den Arbeitskanal des Endoskops vorgeschobene Lasersonde projizierte einen Laserlichtring auf die Bronchialwand, der in allen Bildern gut erkannt werden konnte. Für die bronchoskopische Bestimmung des inneren Durchmessers der Kunststoffröhrchen wurde ein Korrelationskoeffizient von 0,99 ( $p<0,01)$ ermittelt, der Intraclass-Korrelationskoeffiezient (ICC) beträgt 0,97. Slope und Intercept: gemessene Fläche $\left[\mathrm{mm}^{2}\right]=6,47+(0,85 \times$ tatsächliche Fläche). Mithilfe der verzerrungsfreien Video-MakroZoom-Linse wurden tracheale Querschnittsflächen von 197,4 $239,5 \mathrm{~mm}^{2}$ bestimmt. Die korrespondierende Endoskopie ergab Flächeninhalte von 196,6-238,6 mm². Der Korrelationskoeffizient $\mathrm{r}$ betrug $0,88(\mathrm{p}<0,01$, ICC 0,87 , gemessene Fläche $\left[\mathrm{mm}^{2}\right]=19,70+(0,92 \times$ tatsächliche Fläche $)($ Abb. 5) .

Durch die sequenzielle Aufnahme endoskopischer Bilder mithilfe eines kontrollierten Endoskopvorschubs konnte ein dreidimensionaler Datensatz generiert werden. Das Ergebnis der Rekonstruktion und Visualisierung ist in den Abb. 6 u. 7 ersichtlich.

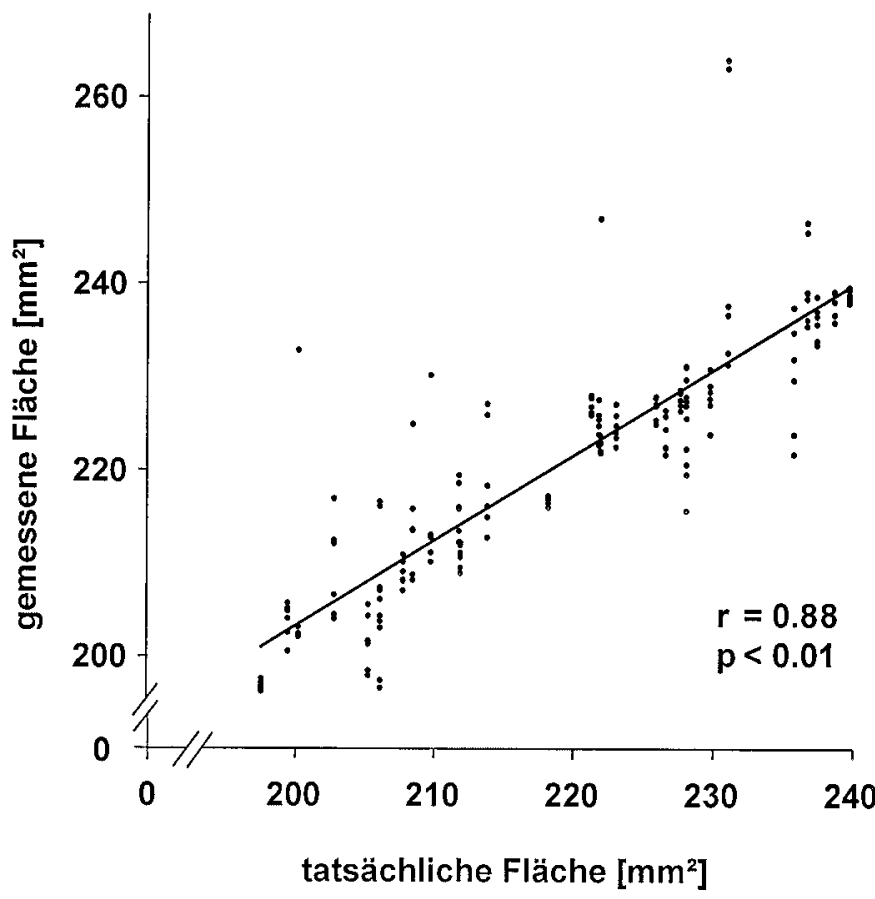

Abb. 5 Abbildung modifiziert nach (16). Vergleich der Ergebnisse der endoskopischen Vermessung mit den tatsächlichen Querschnittsflächen der Schweinetrachea. Die realen Querschnittsflächen wurden anhand von $2 \mathrm{~mm}$ starken Trachealringen mithilfe einer verzerrungsfreien Makro-Zoom-Linse bestimmt.

\section{Diskussion}

Patienten mit bedeutsamen Stenosierungen der Atemwege fallen klinisch häufig mit Stridor und Belastungsdyspnoe aufgrund der erschwerten Atemarbeit auf. Das Spektrum der Methoden, die zur Graduierung von Stenosen der Atemwege eingesetzt wurden, ist vielfältig. Es reicht von den lungenfunktionellen Methoden über die endoskopische und radiologische Diagnostik bis zu experimentellen Verfahren wie z. B. Soundanalysen [1-6].

Mithilfe von lungenfunktionellen Methoden, d.h. durch Messung der dynamischen Atemvolumina bzw. des Atemwegswiderstandes, ist eine funktionelle Beeinträchtigung des Atemwegsquerschnittes abschätzbar. Messungen des Atemwegswiderstandes oder der expiratorischen Flüsse reflektieren die Funktion der Atemwege in toto. Deshalb können die typischen Charakteristika zentraler Stenosen durch Zweiterkrankungen, z.B. eine chronisch obstruktive Lungenerkrankung, so verändert sein, dass eine exakte Schweregradbeurteilung beeinträchtigt wird, mitunter gar unmöglich ist [7]. Eine Alternative könnte hier ein von Waßermann u. Mitarb. vorgestelltes Verfahren sein, dass es mithilfe eines über die Stenose vorgeführten Druckmesskatheters gestattet, lokale Widerstandsmessungen im Tracheobronchialsystem durchzuführen. Dies ist insbesondere wichtig, wenn aufgrund veränderter Strömungsprofile Diskrepanzen zwischen morphologischer und funktioneller Abschätzung der Stenose bestehen [8]. Allen lungenfunktionellen Verfahren ist jedoch gemein, dass es sich um indirekte Methoden zur Abschätzung der Atemwegsquerschnitte handelt. Lediglich die Bronchoskopie gestattet eine direkte Visualisierung der stenotischen Region. Die derzeit akzeptieren Klassifikationssysteme laryngotrachealer 


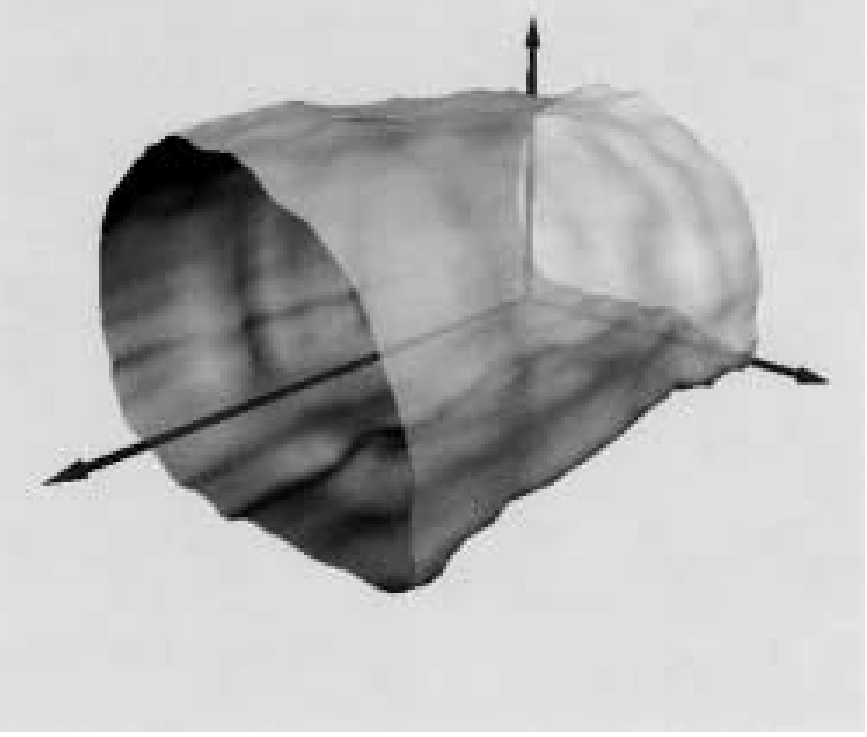

Abb. 6 Dreidimensionale Darstellung eines normalen Trachealabschnittes mithilfe der Visualisierungssoftware Amira.

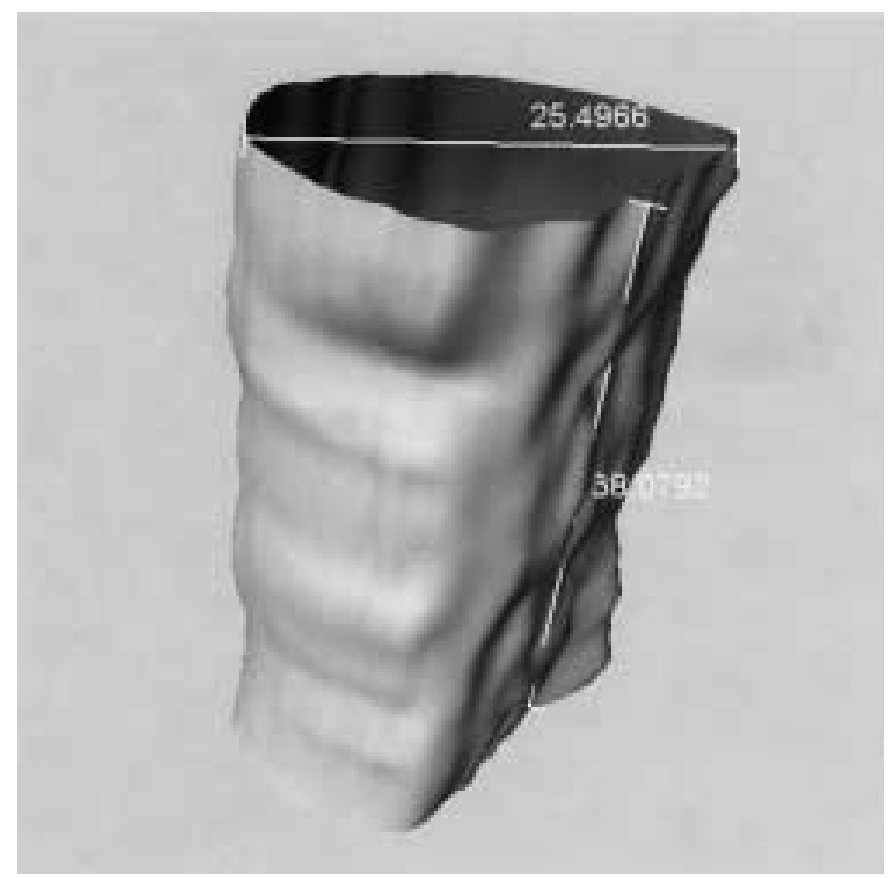

Abb. 7 Von dem dreidimensionalen Objekt können analog zur CTbasierten virtuellen Endoskopie beliebige Ansichten erstellt werden. Die Option der Vermessung könnte z. B. für die Interventionsplanung oder die Nachkontrolle therapeutischer Interventionen von Interesse sein.

Stenosen nach Caffrey oder Cotton [1,2] basieren auf diesem subjektiven Eindruck des Untersuchers. Pathophysiologisch betrachtet sind für den Widerstand einer Stenose jedoch nicht die prozentuale Querschnittsminderung, sondern die absoluten Querschnittsflächen für die Berechnung des Atemwegswiderstandes ausschlaggebend. Für die Vermessung der absoluten Querschnittsflächen haben wir unsere Methodik entwickelt und getestet. Die ermittelten Korrelationen zeigen, dass die Applikation dieses Systems die direkte Vermessung des Atemwegsquerschnittes gestattet. Mithilfe weiterführender Untersuchungen größerer Patientengruppen kann nun die Fragestellung bearbeitet werden, wie sich die Reduktion der absoluten Querschnittsflächen auf den Atemwegswiderstand auswirkt. Sicherlich sind auch hier die Besonderheiten der veränderten Strömungsdynamik (turbulente Flüsse, dynamische Stenosen) von Bedeutung.

Radiologische Untersuchungen, insbesondere die Computertomographie, sind gut geeignet, Atemwegsquerschnitte und die Konfiguration einer Stenose zu erfassen. Ein Nachteil besteht in der Anwendung von Röntgenstrahlen. Andererseits können Stenosen z. B. durch Schleim oder Blutablagerungen in den Atemwegen überschätzt werden. Auch funktionelle Komponenten, entscheidend etwa bei der malazischen Stenose oder dem Tracheobronchialkollaps, kommen nur unzureichend zur Darstellung $[3,9]$. Neuere Entwicklungen wie z.B. die virtuelle Bronchoskopie, basierend auf einem CT-Datensatz, haben die bronchologische Diagnostik bereichert $[9,10]$. Vorteile bestehen z.B. in der Darstellung der Umgebungsstrukturen (z. B. Blutgefäße) und der distal der Stenose gelegenen Atemwege, die sich bei hochgradigen Stenosen einer bronchoskopischen Diagnostik entziehen können. Für eine Vielzahl relevanter Parameter zur Interventionsplanung (dynamischer/fixer Stenoseanteil, Blutungsbereitschaft, Epithelialisierung, Sekretretention, Ödem, Tumorausdehnung) bleibt die endoskopische Diagnostik jedoch das Verfahren der Wahl. Ein Nachteil der Endoskopie besteht bisher in den fehlenden morphometrischen Angaben. Bilder, die mithilfe eines Endoskops erzeugt werden, sind verzerrt: Die Weitwinkellinse bedingt eine „tonnenförmige“ Verzerrung des Objektes. Verschiedene Arbeitsgruppen haben sich mit der Entzerrung endoskopischer Bilder beschäftigt [11-13]. Zur Bestimmung der Atemwegsgröße auf der Grundlage einer Bildentzerrung in vivo ist eine Distanzmessung zwischen Objekt und Objektiv unverzichtbar. Da sich die direkte Distanzmessung nur am Modell realisieren lässt [14], wurden bei Untersuchungen, die der Vermessung endoskopischer Bildinhalte dienten, Körper mit bekannten Größenrelationen (Biopsiezange, Kugeln mit bekanntem Diameter etc.) eingesetzt $[12,13,15]$. Die Praktikabilität dieser Methoden wird durch die Notwendigkeit der Applikation von Referenzkörpern im Bronchialsystem eingeschränkt. Darüber hinaus handelt es sich bei der äußeren Begrenzung eines Atemwegsquerschnittes um eine Zirkumferenz, die unter Zuhilfenahme eines einzigen Referenzkörpers nicht exakt repräsentiert wird. Aus den vorgenannten Gründen erscheint diese Methodik für die Anwendung am Patienten nicht praktikabel.

Die von unserer Arbeitsgruppe vorgeschlagene Lösung durch die Kombination einer Bildentzerrung mit einer lasergestützten Abstandsmessung ermöglicht die direkte Visualisierung des Atemwegsquerschnittes. So lässt sich z.B. das Ausmaß einer Malazie oder eines Tracheobronchialprolaps/-kollaps durch die videoendoskopische Aufnahme des Laserlichtringes während des Atemzyklus bestimmen. Die ermittelten Korrelationskoeffizienten für die Querschnittsflächenbestimmung am Modell $(r=0,99$; $p<0,01$; ICC 0,97$)$ und an der Schweinetrachea $(r=0,88 ; p<0,01$; ICC 0,87$)$ weisen eine gute Übereinstimmung der ermittelten Werte mit den tatsächlichen Querschnitten aus. Die bessere Korrelation bei den Messungen anhand der Kunststoffröhrchen ist 
sicherlich durch die idealen optischen Eigenschaften dieser Testobjekte zu erklären (gute Reflexion durch die glatte Oberfläche und die helle Farbe, gleichmäßige Ausleuchtung und somit gleichmäßig breiter Laserlichtring).

Erste vergleichende Untersuchungen zwischen unserer bronchoskopischen Methode und der CT zeigen hinsichtlich der Bestimmung der kleinsten stenotischen Querschnittsfläche eine gute Übereinstimmung [16]. Größere Serien sind derzeit in Arbeit, um diese Befunde zu validieren. Es stellt sich jedoch die Frage, ob es sich bei der CT um einen verlässlichen „Goldstandard“ handelt.

Bei dem Vergleich o.g. Untersuchungsmethoden zur Beurteilung von Atemwegsstenosen (lungenfunktionelle und radiologische Methoden, Endoskopie) gilt es zu beachten, dass jedes genannte Untersuchungsverfahren nur Teilaspekte der Stenosierung widerspiegelt. Häufig wird man darauf angewiesen sein, diese Methoden komplementär, nicht konkurrierend einzusetzen. Darüber hinaus sind sicherlich auch Aspekte des Gasflusses (turbulente/laminare Strömung, Veränderungen unter der Belastung, dynamische Stenosen) für die funktionelle Relevanz einer Stenosierung von ausschlaggebender Bedeutung.

Technische Nachteile der Vermessungsmethodik bestehen darin, dass der Lichtring während der Untersuchung nicht vollständig im Bild detektiert werden kann, da die Sonde selbst Abschnitte des Ringes verdeckt. Fehler können auch resultieren, wenn die optische Achse des Bronchoskops von der optischen Achse der Lasersonde abweicht. Unter der Voraussetzung, dass es sich bei der wahren Fläche um „A-real“ und „A-ellip“ die durch Abweichung vom Winkel $\alpha=0$ (Winkel zwischen der optischen Achse des Endoskops und der Lasersonde) resultierende falsche elliptische Fläche handelt, gilt:

A-real/A-ellip =x; $x=\cos \alpha$;

Der relative Fehler $(\mathrm{x})$ beträgt somit $(1-\alpha)$. Unter dieser Annahme beträgt der relative Fehler $0,38 \%$ bei einem Winkel von $5^{\circ}$; $1,52 \%$ für $10^{\circ} ; 3,4 \%$ für $15^{\circ} ; 6,03 \%$ für $20^{\circ} ; 9,37 \%$ für $25^{\circ} ; 13,4 \%$ für $30^{\circ}$ und $29,2 \%$ für $45^{\circ}$.

Bei unseren Messungen an Modellen spielte dieser Fehler keine Rolle, da bei der Untersuchung auf der optischen Bank ein Winkel von $0^{\circ}$ gewährleistet war. Nach unseren Erfahrungen ist bei der Messung an Patienten mit einem Winkel von maximal $10-15^{\circ} \mathrm{zu}$ rechnen.

Während der Endoskopie ist es nicht bedeutsam, ob sich die Lasersonde im Zentrum des Bildes oder in der Peripherie befindet.

Bei der Wahl des Behandlungsverfahrens sind nicht nur die Querschnittsflächen, sondern auch die Länge der Stenose und die Konfiguration von Interesse [17]. Neben den funktionellen Konsequenzen, die sich aus der Reduktion des Atemwegsquerschnittes ergeben, determiniert die Konfiguration der Stenosierung hinsichtlich der Längsausdehnung und der Beziehung zum Larynx die Therapieoptionen und das Behandlungsergebnis $[2,17]$.
Unter Anwendung der hier vorgestellten Methode ist es möglich, die Länge einer Stenosierung durch einen kontrollierten Endoskopvorschub (z.B. Markierung an der Zahnreihe) zu erfassen. Die sequenzielle Aufnahme von Schnittbildern in einer definierten Entfernung zueinander gestattet darüber hinaus sogar die Aufnahme eines dreidimensionalen Datensatzes, mit denen sich die endoskopierten Abschnitte rekonstruieren und visualisieren lassen.

Eine kürzlich publizierte Arbeit von Müller u. Mitarb., von denen eine ähnliche Apparatur für die Tracheoskopie entwickelt wurde, dokumentiert ebenfalls eine gute Übereinstimmung der Messwerte mit den tatsächlichen Dimensionen der Trachea. Im Gegensatz zu unserer Methodik registrieren die Autoren zur Bestimmung der Z-Achse (Tiefenausdehnung) nicht den Vorschub des Endoskops, sondern das Ausmaß der Relativbewegung zwischen Lasersonde und Endoskop [18].

Wie in Abb. 6 dargestellt, entsteht durch die Anwendung unserer Methodik ein plastisches Abbild der Stenose. Da der Rekonstruktion die bronchoskopischen Bilder zugrunde liegt, ist es möglich, an dem 3 D Datensatz beliebige zwei- und dreidimensionale Messungen durchzuführen (Abb. 7). Unter anderem eröffnet diese Form der Darstellung die Option einer endobronchialen Tumorvolumenbestimmung und deren Veränderung unter einer Therapie. Darüber hinaus ergeben sich neue Möglichkeiten für virtuelle Interventionen oder z.B. für die individuelle Modellierung von Stents. Die Anwendung dieses Verfahrens in praxi dürfte jedoch derzeit aufgrund der aufwändigen Methodik wenigen Patienten mit komplexen Stenosierungen vorbehalten bleiben.

Die Anwendung des endoskopischen Messsystems ermöglicht eine direkte Dokumentation der Größe und Form der Atemwege. Im Fall von benignen oder malignen Stenosen bereichert diese Technik durch die Möglichkeit der metrischen Erfassung des Stenosierungsgrades die endoskopische Diagnostik. Die Morphometrie gestattet eine bessere Indikationsstellung für eine Intervention und könnte die Planung und Nachkontrolle von Eingriffen an den Atemwegen optimieren. Weitere Untersuchungen größerer Patientenpopulationen sind erforderlich, um den klinischen Stellenwert des vorgestellten Verfahrens in der bronchologischen Diagnostik und Therapie zu validieren.

\section{Literatur}

${ }^{1}$ Cotton RT. Pediatric laryngotracheal stenosis. J Pediatric Surgery 1984 19: $699-704$

${ }^{2}$ McCaffrey TV. Classification of laryngotracheal stenosis. Laryngoscope 1992; 102: $1335-1340$

${ }^{3}$ Brody AS, Kuhn JP, Seidel FG et al. Airway evaluation in children with use of ultrafast CT: pitfalls and recommendations. Radiology 1991; 178: $181-184$

${ }^{4}$ Whyte RI, Quint LE, Kazerooni EA et al. Helical computed tomography for the evaluation of tracheal stenosis. Ann Thorac Surg 1995; 60: 27-30

${ }^{5}$ Vossing M, Wassermann K, Eckel HE et al. Peak flow measurements in patients with laryngeal and tracheal stenoses. A simple and valuable spirometric method. HNO 1995; 43: 70-75

${ }^{6}$ Yonemaru M, Kikuchi K, Mori M et al. Detection of tracheal stenosis by frequency analysis of tracheal sounds. J Appl Physiol 1993; 75: $605-612$ 
${ }^{7}$ Gelb A, Zamel N, Colchen A et al. Physiological studies of tracheobronchial stents in airway obstruction. Am Rev Respir Dis 1992; 146: $1088-1090$

${ }^{8}$ Wassermann K, Koch A, Warschkow A et al. Measuring „in-situ“ central airway resistance in patients with laryngotracheal stenosis. Laryngoscope 1999; 109: 1516 - 1520

${ }^{9}$ Burke AJ, Vining DJ, McGuirt WF et al. Evaluation of airway obstruction using virtual endoscopy. Laryngoscope 2000; 110: 23 - 29

${ }^{10}$ Vining DJ, Liu K, Choplin RH et al. Virtual bronchoscopy. Relationships of virtual reality endobronchial simulations to actual bronchoscopic findings. Chest 1996; 109: 549-553

${ }^{11}$ Smith WE, Vakil N, Maislin SA. Correction of distortion endoscope images. IEEE Trans Med Imaging 1992; 11: 117-122

12 Kouwenhoven E, Mast F, Rijk-Zwikker GL van. Geometrical reconstruction of images obtained with electronic endoscopy. Phys Med Biol 1993; 38: $13-24$

${ }^{13}$ Vakil N, Smith W, Bourgeois K et al. Endoscopic measurement of lesion size: improved accuracy with image processing. Gastrointest Endosc 1994; 40: $178-183$

${ }^{14}$ Santos MC, Strande L, Doolin EJ. Airway measurement using morphometric analysis. Ann Otol Rhinol Laryngol 1995; 104: 835-838

${ }^{15}$ Forkert L, Watanabe H, Sutherland K et al. Quantitative videobronchoscopy: a new technique to assess airway caliber. Am J Respir Crit Care Med 1996; 154: 1794-1803

${ }^{16}$ Dörffel WV, Fietze I, Hentschel D et al. A new bronchoscopic method to measure airway size. Eur Respir J 1999; 14: 783-788

17 Deutsche Gesellschaft für Pneumologie - Arbeitsgruppe „Qualitätssicherung in der Bronchologie“. Empfehlungen zur bronchoskopischen Behandlung tracheobronchialer Verschlüsse, Stenosen und muraler maligner Tumoren. Pneumologie 1998; 52: 243-248

${ }^{18}$ Müller A, Schubert M, Begleites E. Noncontact three-dimensional laser measuring device for tracheoscopy. Ann Otol Rhinol Laryngol 2002; 111: $821-827$

\section{Erratum}

Ulmer, WT et al.: Die Lungenfunktion: State-of-the-Art und neue Ergebnisse, Pneumologie 2003; 57: 400-401

Hier wurde der Name des Zweitautors falsch abgedruckt sowie die Literatur vergessen. Der Zweitautor heißt richtig T. Schäfer.

\section{Literatur}

${ }^{1}$ Hutchinson J. Lecture on vital statistics, embracing an account of a new instrument for detecting the presence of disease of the system. Lancet 1844: 567-570, $594-596$

${ }^{2}$ Hutchinson J. On capacity of lungs and on respiratory functions with view of establishing precise and easy method of detecting disease by spirometer. Trans med-chir Soc London 1846; 29: 137

${ }^{3}$ Hankinson JL. Does Poor Quality Render it Inpractical? Chest 1999; 116/2: $276-277$

${ }^{4}$ Schäfer T, Happel A, Islamova S, Schläfke ME, Ulmer WT. Zur Stabilität lungenfunktionsanalytischer Parameter: Spirometrie, Bodyplethysmographie und Mundverschlußdruck (PO.1). Pneumologie 2002; 56: $679-683$

Der Verlag bittet dieses Versehen zu entschuldigen. 